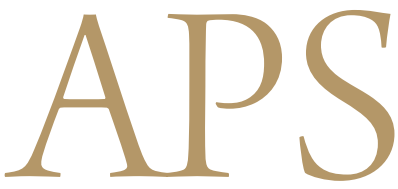

Archives of Plastic Surgery

\title{
Outcomes of Surgical Management of Xanthelasma Palpebrarum
}

\author{
Hoon Young Lee ${ }^{1}$, Ung Sik Jin ${ }^{1}$, Kyung Won Minn ${ }^{1}$, Young-Oh Park \\ ${ }^{1}$ Department of Plastic and Reconstructive Surgery, Seoul National University College of Medicine, Seoul; ${ }^{2}$ Ever Plastic Surgery Clinic, Seoul, \\ Korea
}

Background Xanthelasma palpebrarum (XP) is a benign disorder manifesting as yellowish cholesterol-laden plaques on the eyelids. This paper presents the outcomes in patients with XP who have undergone surgical excision as the main modality of treatment.

Methods A retrospective review of patients who received surgery for xanthelasma palpebrarum from March 2007 to March 2011 was conducted. Patients were classified into four grades according to the location and extent of the lesion, with grade I being the mildest and grade IV being the most diffuse. Simple excision was performed in grade I and II lesions, while local flaps and skin grafts were performed in the more advanced grades.

Results Ninety-five cases from March 2007 to March 2011 were included in this study. 66 cases $(70 \%)$, were treated by simple excision. Twenty-four cases (25\%) and 5 cases (5\%) were treated by simple excision in combination with or without local flaps and skin grafts. In approximately $1 / 4$ of the patients, orbicularis oris muscle involvement was observed. 4 patients (4.2\%) developed scar contracture postoperatively, which required a secondary procedure. Recurrence was reported in 3 patients (3.1\%). Otherwise. There were no other reports of major complications or disfigurement.

Conclusions We found that for lesions involving the deep dermis and/or muscle, surgical excision was the most appropriate therapeutic option.

Keywords Xanthomatosis / Foam cells / Reconstructive surgical procedures / Eyelids
Correspondence: Ung Sik Jin Department of Plastic and Reconstructive Surgery, Seoul National University College of Medicine, 101 Daehang-ro, Jongno-gu, Seoul 110-744, Korea

Tel: +82-2-2072-2375

Fax: +82-2-3675-7792

E-mail: usj1011@snu.ac.kr

Received: 7 Feb 2013 • Revised: 17 Mar 2013 Accepted: 27 May 2013

pISSN: 2234-6163 • elSSN: 2234-6171 • http://dx.doi.org/10.5999/aps.2013.40.4.380 • Arch Plast Surg 2013;40:380-386

This article was presented at the 69th Congress of the Korean Society of Plastic and Reconstructive Surgeons on November 11-13, 2011 in Seoul, Korea.

No potential conflict of interest relevant to this article was reported.

\section{INTRODUCTION}

Xanthelasma palpebrarum is a common benign disorder manifesting as yellowish plaques on the eyelids, resulting from the deposition of cholesterol laden histiocytes. Xanthelasma palpabrarum is the most common cutaneous xanthoma. Histologically, xanthelasmas are composed of xanthoma cells, or foam cells, which are typically found in the middle and superficial layers of the dermis in perivascular and periadnexal locations, with evidence of concurring fibrosis and inflammation [1].

Xanthelasma often indicates an underlying plasma lipid disorder and has been identified as a marker for increased risk of atherosclerosis inferred from associated lipoprotein and apolipoprotein abnormalities [2]. Hyperlipidemia is reported to occur in approximately 50 percent of patients with xanthelasma [1]. However, xanthelasma can occur in patients with a normolipidemic profile [2].

These lesions rarely cause functional problems such as ob- 
structed vision, and treatment is usually sought due to aesthetic compromises of the periorbital area caused by fibrotic plaques. Numerous treatment options, both local and systemic, have been reviewed in the literature, and include surgical resection [3]; ablation with various lasers such as carbon dioxide [4], erbium-YAG [5], pulsed dye [6], and Nd-YAG [7]; tricholoracetic acid peels $[6]$; and treatment of the underlying medical condition $[1,2,8]$.

This paper presents the outcomes in patients presenting with xanthelasma palpebrarum who underwent surgical excision as the main modality of treatment. We performed a retrospective review of patient records from 2007 to 2011 of xanthelasma palpebrarum at our clinic. The purpose of our study was to further define the clinicopathologic factors of xanthelasma palpebrarum and the role of surgery in the treatment of xanthelasma by evaluating recurrence and complications.

\section{METHODS}

A retrospective review of 95 patients (male:female, 2:3), who underwent surgery for xanthelasma palpebrarum from March 2007 to March 2011, was conducted. The diagnosis of xanthelas- ma palpebrarum was based on physical presentation and clinical examination. A lipid profile was obtained for all patients through standard laboratory blood tests. Preoperative biopsies were obtained to determine the lesion depth.

The patients were classified into four grades according to the location and extent of the lesion. Grade I comprised patients who presented with lesions on the upper eyelids only. Grade II comprised patients whose lesions extended to the medial canthal area. Grade III comprised patients with lesions on the medial side of the upper and lower eyelids. Grade IV comprised patients whose lesions were diffuse, with involvement of the medial and lateral sides of the upper and lower eyelids (Fig. 1). The height of the lesions was also noted.

Surgical excision was performed in conjunction with blepharoplasty, medial epicanthoplasty, "uncapping surgery" where the lesion is "uncapped" and the cholesterol deposit is removed in one piece followed by closure1, local skin advancement flaps or orbicularis oculi muscle flaps, and skin grafts. The results of the surgical modality used in each aforementioned classification were assessed for recurrence and complications. Patient characteristics according to the lesion grade are summarized in Table 1.

\section{Fig. 1. Clinical presentation of xanthelasma palpebrarum according to grade}

(A) Grade I lesion, involving only the upper eyelids. (B) Grade II lesion, extending to the medial canthal area. (C) Grade III lesion, involving the medial side of the upper and lower eyelids. (D) Grade IV lesion, diffuse involvement of the medial and lateral side of the upper and lower eyelids.
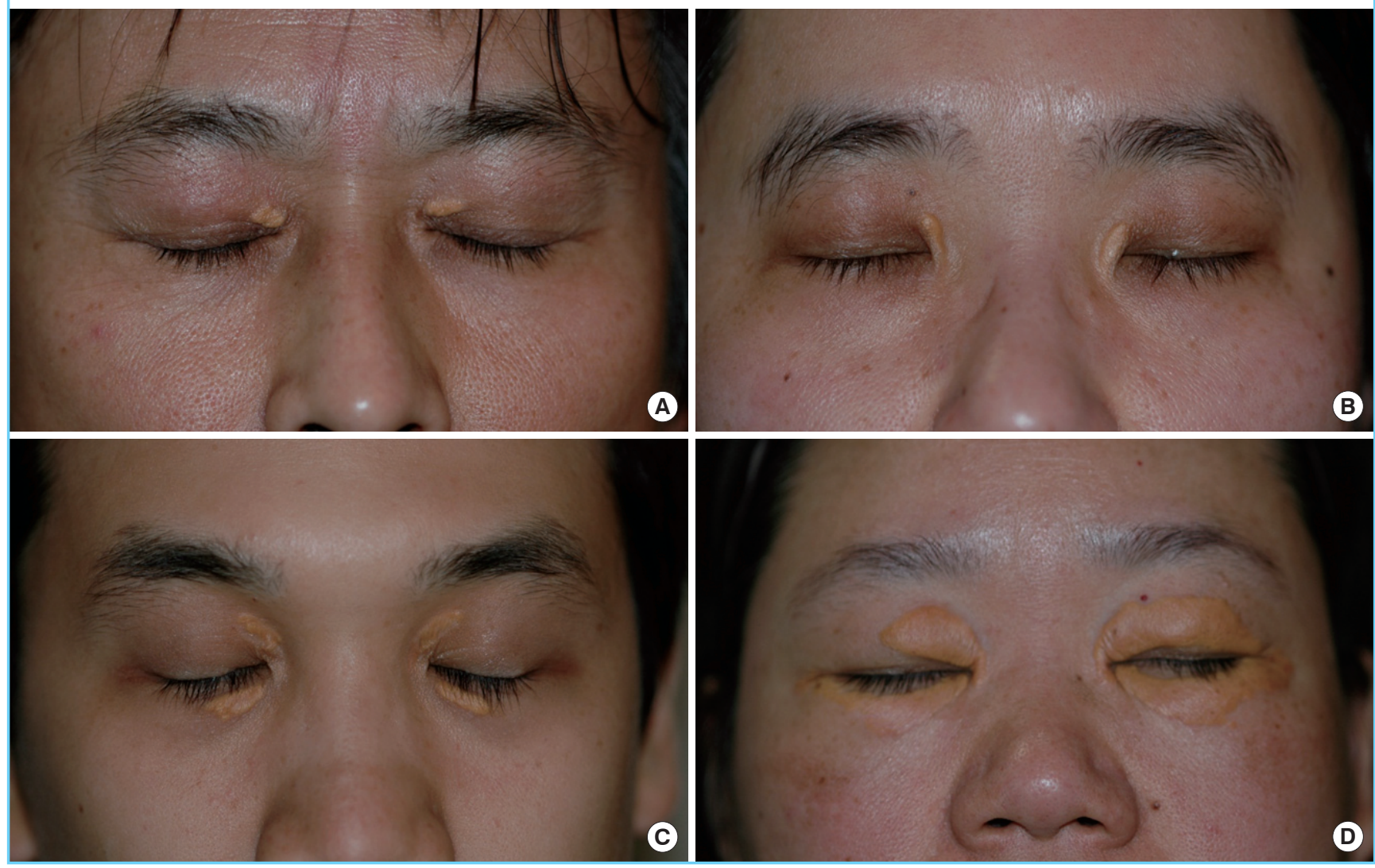
Table 1. Patient characteristics and surgical methods

\begin{tabular}{lccccc}
\hline Lesion grade & $\begin{array}{c}\text { Patient } \\
\text { No. (\%) }\end{array}$ & Surgical methods & Accompanying surgery & Minor complications & Recurrence \\
\hline Grade I & $33(35)$ & Simple excision & Blepharoplasty & None & None \\
Grade II & $33(35)$ & Simple excision & Blepharoplasty, medial epicanthoplasty & None \\
Grade III & $24(25)$ & $\begin{array}{c}\text { Simple excision, uncapping surgery, } \\
\text { skin flap advancement, orbicularis } \\
\text { muscle flap, skin graft }\end{array}$ & Blepharoplasty, medial epicanthoplasty & Scar contracture $2(2.1 \%)$ & $2(2.1 \%)$ \\
Grade IV & $5(5)$ & Blepharoplasty, medial epicanthoplasty & Scar contracture 2(2.1\%) \\
Total & $95(100)$ & & & None \\
\hline
\end{tabular}

\section{Fig. 2. Grade IV diffuse lesion}

$(A, B)$ Preoperative photo of a grade IV diffuse lesion involving the medial and lateral sides of both upper and lower eyelids. (C, D) Postoperative photo at 1 year after surgical excision by upper blepharoplasty and lower lid orbicularis myocutaneous advancement flap.
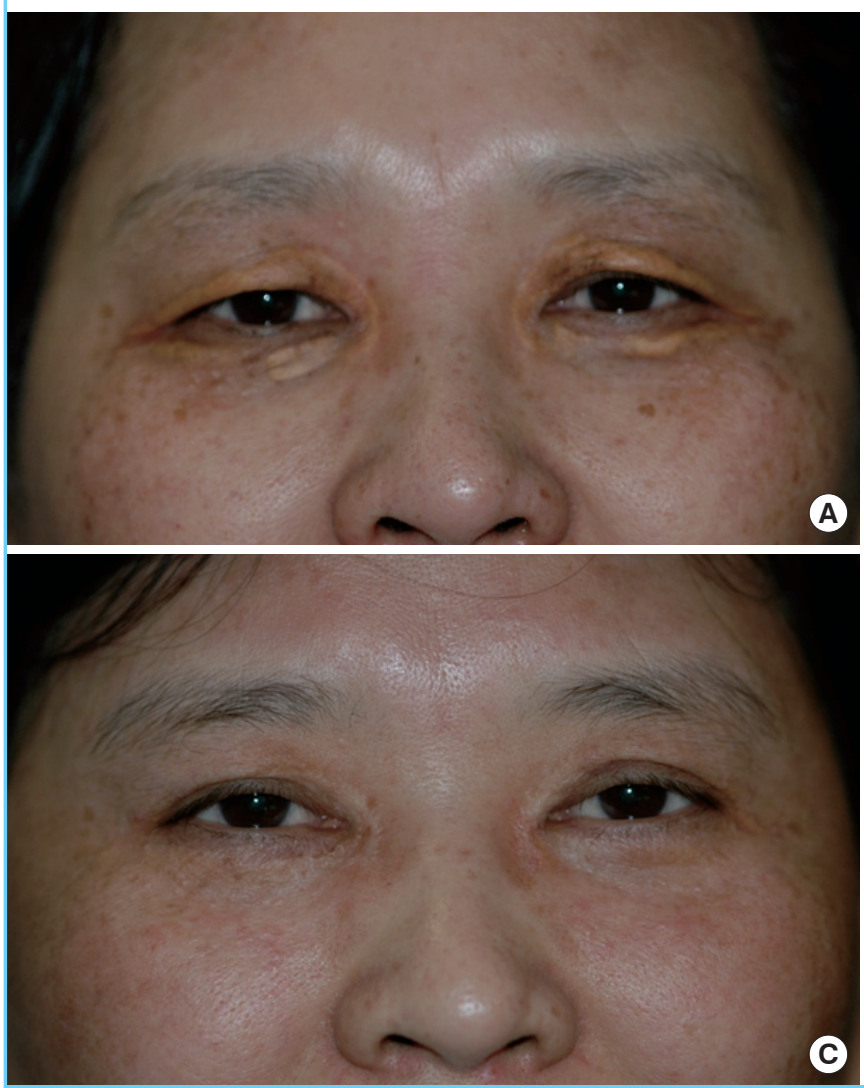

\section{RESULTS}

A total of 95 cases from March 2007 to March 2011 were included in this study. The mean age of the patients was 56 years, and the male-to-female ratio of the patient population was approximately $2: 3$. The patient population who presented with an abnormal lipid profile was $52 \%$. Preoperative biopsies were performed in all of the cases.

Simple excision was performed for the grade I and II lesions, while local flaps and skin grafts were performed in the more advanced grades of the disease. Sixty-six cases (70\%), generally
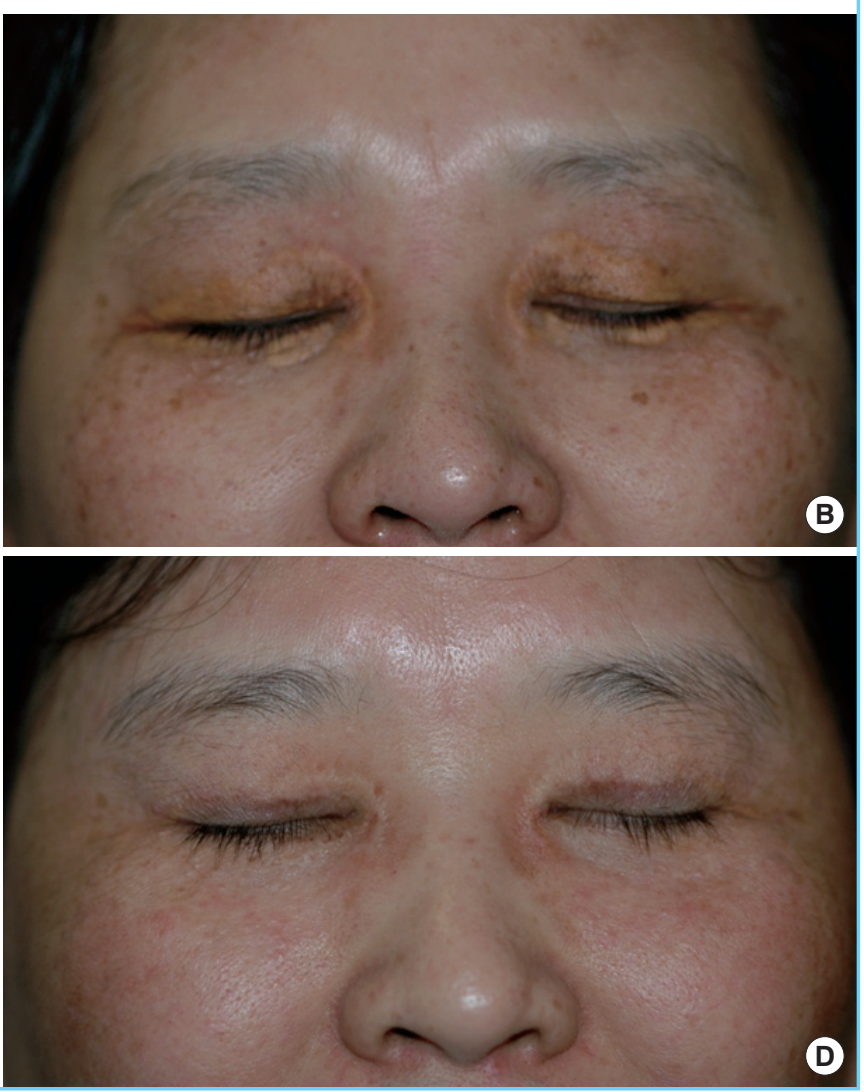

grade I or II lesions, were treated by simple excision with blepharoplasty design. In 33 patients (35\%) who presented with grade I lesions, limited to the superficial dermis, simple excision was performed. In 33 patients (35\%) who presented with grade II lesions extending to the medial canthal area, concomitant medial epicanthoplasty was necessary.

The surgical modality for more advanced stages of the disease with involvement of the medial side of the upper and lower eyelids (grade III), and with diffuse involvement of the medial and lateral sides of both upper and lower eyelids (grade IV) was simple excision by blepharoplasty in combination with or with- 


\section{Fig. 3. Grade IV diffuse lesion}

(A, B) Preoperative photo of a grade IV diffuse lesion involving the medial and lateral sides of both upper and lower eyelids. (C, D) Postoperative photo at 1 year after surgical excision by upper blepharoplasty, lower lid orbicularis myocutaneous advancement flap, and full-thickness skin graft with excised skin from blepharoplasty. Postoperative photos show complete excision of the lesions with relatively minimal scarring and no sign of recurrence. The patient expressed satisfaction with the overall aesthetic result.
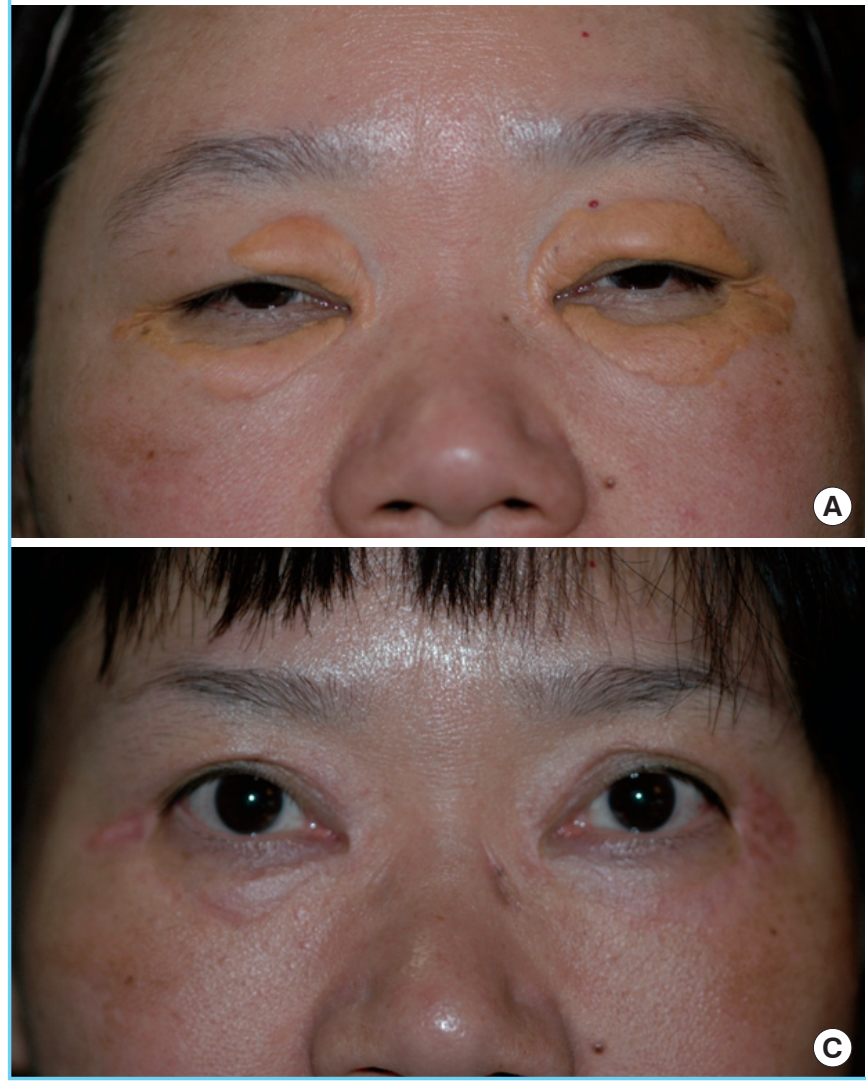
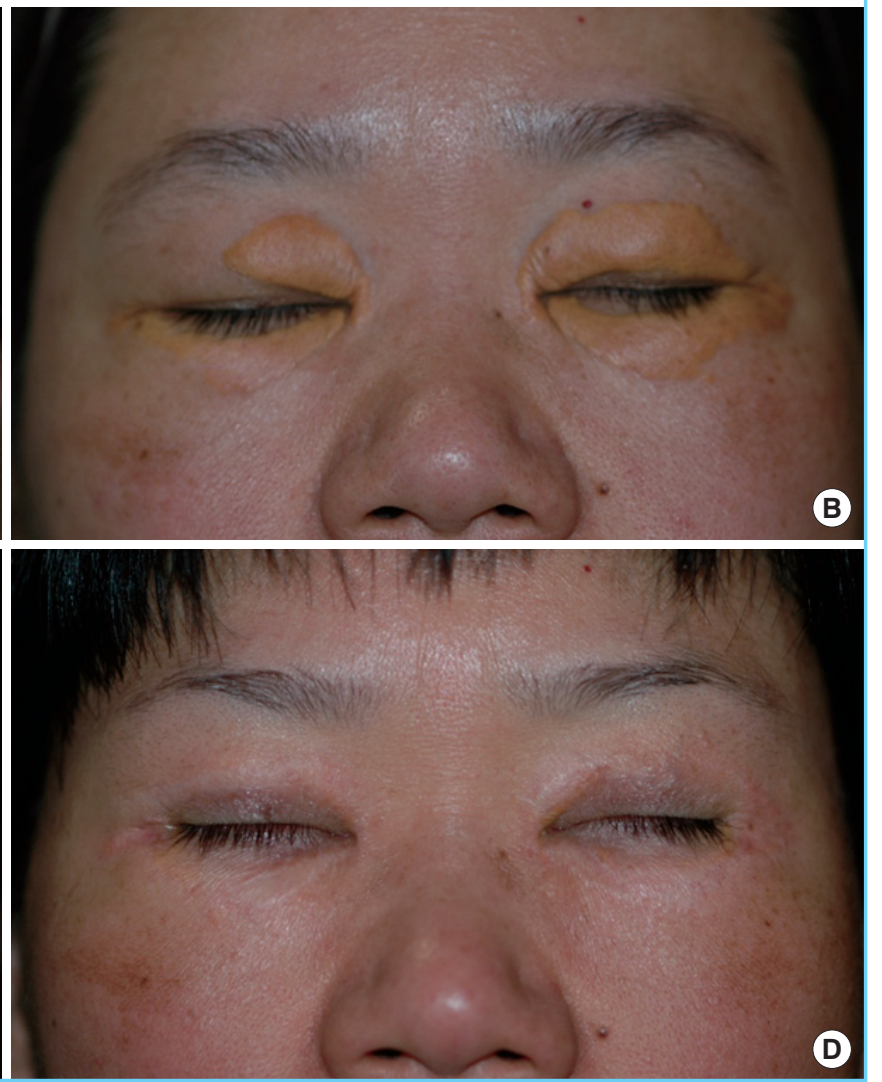

out local flaps and skin grafts. Skin grafts were performed using the excised normal skin from the blepharoplasty. Twenty-four cases $(25 \%)$ were grade III lesions, and 5 cases (5\%) were grade IV lesions (Figs. 2, 3).

In our study, 7 cases of grade I lesions and 6 cases of grade II lesions showed involvement of the orbicularis oculi muscle, and the affected musculature was completely resected. Grade III and grade IV lesions generally showed muscle involvement, and to provide tension-free closure after excision of these diffuse lesions, concomitant muscle excision was compulsory. When the size of the lesions exceeded $5 \mathrm{~mm}$ in height, surgical management, regardless of the lesion depth, was preferred.

Four patients (4.2\%) developed scar contracture postoperatively, which required a secondary procedure such as scar revision or medial epicanthoplasty. During 1 year of postoperative follow-up, lesion recurrence was reported in only 3 patients (3.1\%). Otherwise, there were no other major complications or disfigurement reported in this study.

\section{DISCUSSION}

Xanthelasma palpebrarum manifests as yellowish fibrotic plaques on the eyelids, resulting from the deposition of cholesterol laden histiocytes in the dermis. Histologically, xanthelasmas are composed of xanthoma cells, or foam cells, which are typically found within the middle and superficial layers of the dermis in perivascular and periadnexal locations, with evidence of concurrent fibrosis and inflammation [1,2]. In some cases, xanthelasma can infiltrate the muscle layer and muscle resection is required for complete excision of the lesion [9]. In this study, muscular infiltration was seen in approximately $25 \%$ of patients (Fig. 4). Incomplete excision of the lesion leads to a higher incidence of recurrence.

Numerous treatment options, both local and systemic, have been reviewed in the literature, including surgical resection [3]; ablation with various lasers such as carbon dioxide [4], erbiumYAG [5], pulsed dye [6], and Nd-YAG [7]; tricholoracetic acid peels [6]; and treatment of the underlying medical condition $[1,2,8]$. However, each method of treatment is associated with 


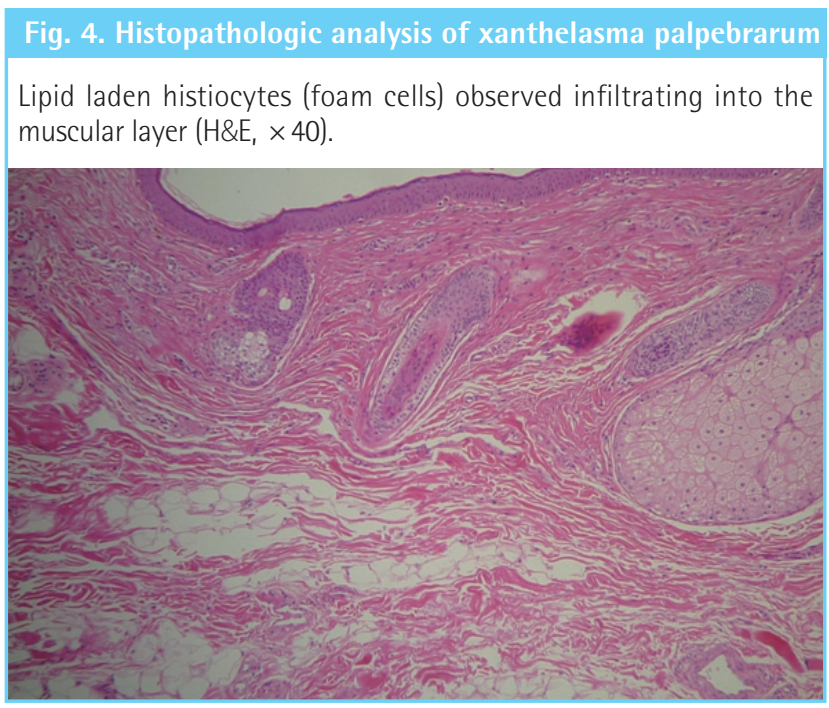

limitations and complications such as persistent erythema, recurrence, ectropion, scarring, and hypo- or hyperpigmentation [10]. Mendelson and Masson [3] found that $40 \%$ of patients had recurrence after primary surgical excision, $60 \%$ after secondary excision, and $80 \%$ when all four eyelids were involved. Mendelson and Masson did not mention lesion extension in regard to depth, and may not have acquired an adequate resection depth margin in the surgical treatment of xanthelasma palpebrarum, accounting for their relatively high recurrence rate. However, this was not discussed in their paper, and it is difficult to draw conclusions.

Treatment with various lasers for xanthelasma palpebrarum has been reviewed in the literature with extensive variations in outcome $[4,5,7,10-12]$. At our center, laser therapy as well as surgical excision has been utilized as a therapeutic module with satisfactory results. Primary laser therapy with an ablative carbon dioxide laser or erbium-YAG was reserved for treatment only in small aberrant lesions and lesions involving the superficial dermis. However, because the focus of our paper is on the surgical results of xanthelasma palpebrarum, the results analysis of laser therapy for superficial lesions has not been included in this study. Fusade [7] suggested that a 1,064-nm Q-switched Nd:YAG laser is a valuable treatment option for xanthelasma, while Karsai et al. [13] reported that Q-switched Nd:YAG laser treatment of xanthelasma is not recommended. Argon laser photocoagulation treatment was found by Hintschich1 to have a high recurrence rate [1]. Raulin et al. [4] reviewed their experiences with ultrapulsed carbon dioxide laser and reported that treatment in the early stages of xanthelasma development was crucial in order to prevent recurrence. Lieb et al. [14] revealed that wound healing with carbon dioxide laser was significantly slower due to a larger thermal necrosis zone. Tricholoracetic acid in the man- agement of xanthelasma was found to be effective for treatment of smaller lesions, but repeat procedures were common and pigmentation and scarring were common cosmetic concerns [6]. Mittelviefhaus et al. [9] found through histologic specimens that in a large number of cases, the depth of tissue invasion in xanthelasma infiltrates through the entire dermis, reaching the stratum muscular, and even invading into this layer; these lesions, they proposed, should not be treated by lasers, but should be surgically excised [8].

Recurrence of xanthelasma palpebrarum is a common problem [1]. There are numerous treatment options, both local and systemic, for this condition. In lesions presented at our clinic that were confined to the superficial dermis, less than $5 \mathrm{~mm}$ in height, and with an onset of less than one year, laser ablation was a treatment option, and in some cases this treatment alone sufficed. However, when lesions involved deeper layers, recurrence rates were higher, and this is attributable to the fact that lasers may not provide a clean resection margin due to a lack of technical skill or penetration. Furthermore, superficial ablation of lesions may require multiple therapy sessions. When lesions involve the deep dermis or infiltrate the underlying musculature, surgical excision is the mainstay of treatment. Through our experience, we have found that for longstanding lesions with an onset exceeding a year, or large lesions extending beyond $5 \mathrm{~mm}$ in height, local flaps and skin grafts may be necessary to preserve the aesthetic continuity of the eyelids, regardless of the lesion depth. Also, simple excision by blepharoplasty may provide a more aesthetically pleasing result after surgery. It is generally known that only small eyelid defects that are less than $5 \mathrm{~mm}$ are amenable to healing by secondary intention [15]. Lasers and other nonsurgical treatment options ultimately result in a partial skin defect of the eyelid. Thus, to prevent eyelid deformation and to achieve a more tolerable scar, surgical management is recommended. A clinically large lesion (i.e., lesions greater than $5 \mathrm{~mm}$ in height), regardless of depth, when treated with lasers, resulted in a more blatant secondary deformity and was more susceptible to skin discoloration, scarring, and recurrence.

Our study presents only the outcomes of surgical cases of xanthelasma palpebrarum. In patients with lesions involving only the superficial dermis, a multitude of less invasive treatment options exists that may provide satisfactory outcomes. In patients who were hesitant to undergo invasive approaches who presented with superficial lesions at our clinic, lasers have also provided satisfactory outcomes. We recommended surgical treatment to patients who we predicted would benefit aesthetically from a surgical approach, such as those with diffuse lesions, dermatochalasia, baggy lower eyelids, or skin laxity. Thus, in the lesions that are confined to the superficial dermis, treatment should 
be individualized for patient preference and characteristics. We acknowledge that this is a limitation of our study and a comparative study of different modalities of treatment with results analysis will provide further credibility to our conclusion.

Observational studies have linked the presence of xanthelasma palpebrarum with conditions such as hyperlipidemia, dyslipoproteinemia, hypertension, and underlying cardiovascular disease such as atherosclerosis. Underlying medical conditions such as hypothyroidism, renal syndromes, and cirrhosis have also been reported in the differential diagnosis for these cutaneous lesions. Histological analysis has revealed similarities in the ultrastructure of xanthelasma and atheroma formation and several data suggest that there may be an association with low high density lipoproteinqq cholesterol levels and other lipoprotein and apolipoprotein abnormalities [2]. Furthermore, in the pathogenesis of xanthelasma, abnormal lipid metabolism seems to play a crucial role. However, the appearance of xanthelasmas in patients with a normolipidemic profile and studies that conclude no relationship between plasma lipoprotein abnormalities and xanthelasma suggest that the formation of xanthelasmas may be influenced by, but not indicative of, underlying disease $[2,3,8]$. In our study, only $52 \%$ of patients presented with an abnormal lipid profile. Whether or not restriction of fat intake may correlate with regression of xanthelasma remains relatively obscure. Although xanthelasmas are not definitely determinate cutaneous markers of atherosclerosis or other underlying conditions, inconsistency in the results in the pathogenesis of xanthelasma cannot be ignored $[1,2,8]$. At our clinic, it was recommended that patients with underlying medical conditions or those who presented with an abnormal lipid profile see an internal medicine specialist for a further work-up and treatment, if necessary.

We have developed a treatment algorithm for xanthelasma, taking into account patient characteristics and the size and location of the lesion. Xanthelasma palpebrarum has been correlated in

\section{Fig. 5. Proposed algorithm for the treatment of xanthelasma palpebrarum}

The size and location of the lesion should be taken into account in the treatment of xanthelasma palpebrarum. In lesions involving the deep dermis and/or muscle, surgical excision is the most appropriate therapeutic option. LDL, low density lipoprotein; IHD, ischemic heart disease. ${ }^{\text {a) }}$ refer to internal medicine specialist.

Laboratory assessment

Therapeutic control

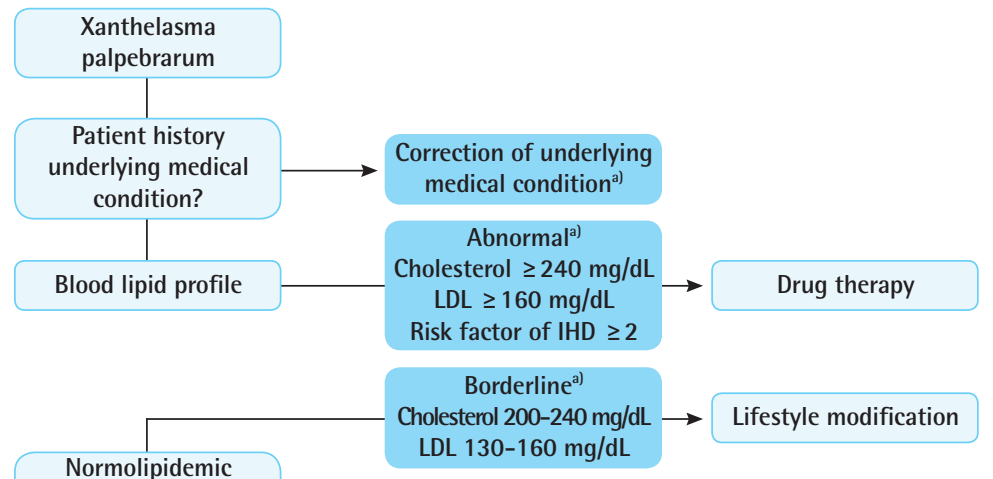

Normolipidemic no underlying medical condition or recalcitrant to therapeutic control

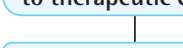

Correction of underlying

\section{Abnormal ${ }^{\mathrm{a}}$}

Cholesterol $\geq 240 \mathrm{mg} / \mathrm{dL} \rightarrow$ Drug therap LDL $130-160 \mathrm{mg} / \mathrm{dL}$
Grade I, II, III (lesion depth: limited to superficial dermis)

Lesion height $\leq 5 \mathrm{~mm}$

Onset $\leq 1 \mathrm{yr}$

Patient preference
Laser

\section{Biopsy}

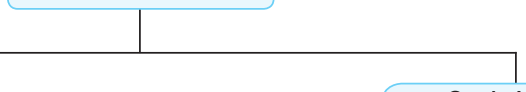

Grade I, II, III

(lesion depth: deep dermis-muscle)

Skin laxity, Blepharochalasia Need of aesthetic refinement

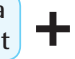

Grade IV

Lesion height $\geq 5 \mathrm{~mm}$

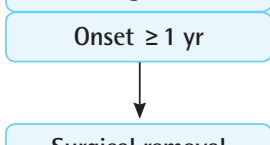

Simple excision with or without blepharoplasty

Skin or orbicularis M. advancement flap

Surgical removal 
some cases with the presence of an underlying medical condition, and therefore, systemic treatment of the underlying medical condition with lifestyle modification or pharmaceutical therapy can be attempted before or in conjunction with surgical excision. In lesions with only a superficial extent, laser therapy may suffice, but in some cases, surgery may provide a more aesthetic result. In such cases, treatment should be individualized in each case. In conclusion, among the numerous treatment options for xanthelasma, we have found that for lesions involving the deep dermis and/or muscle, surgical excision was the most appropriate therapeutic option (Fig. 5). In diffuse lesions (grades III, IV), excision in conjunction with local flaps or skin grafts provided aesthetic reconstruction with a low incidence of recurrence.

\section{REFERENCES}

1. Rohrich RJ, Janis JE, Pownell PH. Xanthelasma palpebrarum: a review and current management principles. Plast Reconstr Surg 2002;110:1310-4.

2. Bergman $\mathrm{R}$. The pathogenesis and clinical significance of xanthelasma palpebrarum. J Am Acad Dermatol 1994;30: 236-42.

3. Mendelson BC, Masson JK. Xanthelasma: follow-up on results after surgical excision. Plast Reconstr Surg 1976;58: 535-8.

4. Raulin C, Schoenermark MP, Werner S, et al. Xanthelasma palpebrarum: treatment with the ultrapulsed CO2 laser. Lasers Surg Med 1999;24:122-7.

5. Borelli C, Kaudewitz P. Xanthelasma palpebrarum: treatment with the erbium:YAG laser. Lasers Surg Med 2001;29: 260-4.
6. Cannon PS, Ajit R, Leatherbarrow B. Efficacy of trichloroacetic acid (95\%) in the management of xanthelasma palpebrarum. Clin Exp Dermatol 2010;35:845-8.

7. Fusade T. Treatment of xanthelasma palpebrarum by $1064-$ nm Q-switched Nd:YAG laser: a study of 11 cases. Br J Dermatol 2008;158:84-7.

8. Ozdol S, Sahin S, Tokgozoglu L. Xanthelasma palpebrarum and its relation to atherosclerotic risk factors and lipoprotein (a). Int J Dermatol 2008;47:785-9.

9. Mittelviefhaus $\mathrm{H}$, Kreusser C, Bohringer D, et al. The underestimated depth of tissue invasion of xanthelasma: a histological study. Klin Monbl Augenheilkd 2011;228:14-8.

10. Park EJ, Youn SH, Cho EB, et al. Xanthelasma palpebrarum treatment with a 1,450-nm-diode laser. Dermatol Surg 2011; 37:791-6.

11. Hintschich C. Argon laser coagulation of xanthelasmas. Ophthalmologe 1995;92:858-61.

12. Katz TM, Goldberg LH, Friedman PM. Fractional photothermolysis: a new therapeutic modality for xanthelasma. Arch Dermatol 2009; 145:1091-4.

13. Karsai S, Schmitt L, Raulin C. Is Q-switched neodymiumdoped yttrium aluminium garnet laser an effective approach to treat xanthelasma palpebrarum? Results from a clinical study of 76 cases. Dermatol Surg 2009;35:1962-9.

14. Lieb WE, Klink T, Munnich S. CO2 and erbium YAG laser in eyelid surgery. A comparison. Ophthalmologe 2000;97: 835-41.

15. Lowry JC, Bartley GB, Garrity JA. The role of second-intention healing in periocular reconstruction. Ophthal Plast Reconstr Surg 1997;13:174-88. 